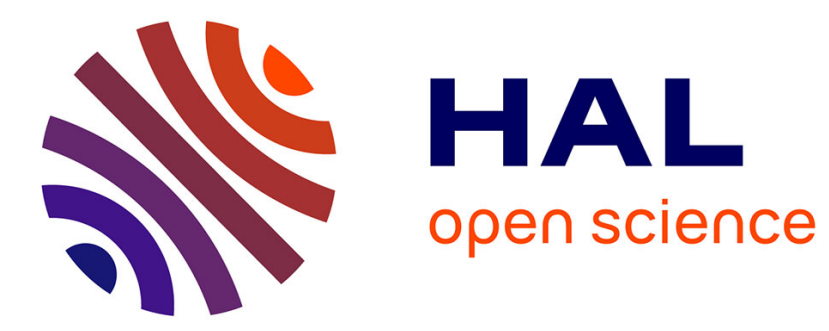

\title{
Stability analysis of a singularly perturbed coupled ODE-PDE system
}

Ying Tang, Christophe Prieur, Antoine Girard

\section{To cite this version:}

Ying Tang, Christophe Prieur, Antoine Girard. Stability analysis of a singularly perturbed coupled ODE-PDE system. CDC 2015 - 54th IEEE Conference on Decision and Control, Dec 2015, Osaka, Japan. 10.1109/CDC.2015.7402936 . hal-01276239

\section{HAL Id: hal-01276239 \\ https://hal.science/hal-01276239}

Submitted on 19 Feb 2016

HAL is a multi-disciplinary open access archive for the deposit and dissemination of scientific research documents, whether they are published or not. The documents may come from teaching and research institutions in France or abroad, or from public or private research centers.
L'archive ouverte pluridisciplinaire HAL, est destinée au dépôt et à la diffusion de documents scientifiques de niveau recherche, publiés ou non, émanant des établissements d'enseignement et de recherche français ou étrangers, des laboratoires publics ou privés. 


\title{
Stability analysis of a singularly perturbed coupled ODE-PDE system
}

\author{
Ying TANG, Christophe PRIEUR and Antoine GIRARD
}

\begin{abstract}
This paper is concerned with a coupled ODEPDE system with two time scales modeled by a perturbation parameter. Firstly, the perturbation parameter is introduced into the PDE system. We show that the stability of the full system is guaranteed by the stability of the reduced and the boundary-layer subsystems. A numerical simulation on a gas flow transport model is used to illustrate the first result. Secondly, an example is used to show that the full system can be unstable even though both subsystems are stable when the perturbation parameter is introduced into the ODE system.
\end{abstract}

\section{INTRODUCTION}

The gas flow transport is a phenomenon usually found in industrial applications. For example, control of the fraction of fresh air in engines of automobiles in [24] or the ventilation systems in mines [25]. It is of great interest for both energy supply and control of such system. The dynamics of the gas flow system have been modeled by partial differential equations (PDEs) in many research works, for instance [6] and [10].

Lyapunov method, which is commonly used for the stability analysis of dynamical systems, has been employed in many works for singularly perturbed ordinary differential equations system, see for instance [14], [4], [12], [5] and [19]. It is also a powerful tool for the stability analysis of partial differential equations. In [7] a strict $H^{2}$-norm Lyapunov function has been constructed to analyze the stability of solutions to a system of two hyperbolic conservation laws around equilibrium. The stability of one-dimensional $n \times n$ nonlinear hyperbolic systems has also been considered in [8]. In the work of [13], it has been concerned with $H^{2}$ stabilization of the Isothermal Euler equations. The stability of a class of singularly perturbed hyperbolic systems has been studied in [23], [22].

In many research works, backstepping approach is used to stabilize ordinary differential equation - partial differential equation (ODE-PDE) systems. For example, a coupled firstorder hyperbolic PDE and second-order (in space) ODE has been stabilized by this approach in [18]. In [17], predictorlike feedback laws and observers have been designed for a diffusion PDE (heat equation) and linear time invariant (LTI) ODE in cascade. A cascade of second order (in time) PDE and ODE has been studied in [16]. Lyapunov technique is also used to analyze the stability for such systems. Indeed in [11], a strict Lyapunov function has been used to prove

Y. TANG and C. PRIEUR are with Department of Automatic Control, Gipsa-lab, 11 rue des Mathematiques, BP 46, 38402 Saint Martin d'Heres, France, ying.tang@gipsa-lab.fr, christophe.prieuregipsa-lab.fr.

A. GIRARD is with Laboratoire Jean Kuntzmann, Université de Grenoble, B.P. 53, 38041 Grenoble, France. antoine.girardeimag. fr. the stability of a hyperbolic system with the integral actions at the boundary, which is a kind of ODE-PDE system. An open-loop unstable hyperbolic system has been stabilized by PI boundary controller, which is proved in frequency domain in [2].

The motivation of this work is from the experimental setup (Figure 1) which can be used to control the gas flow.

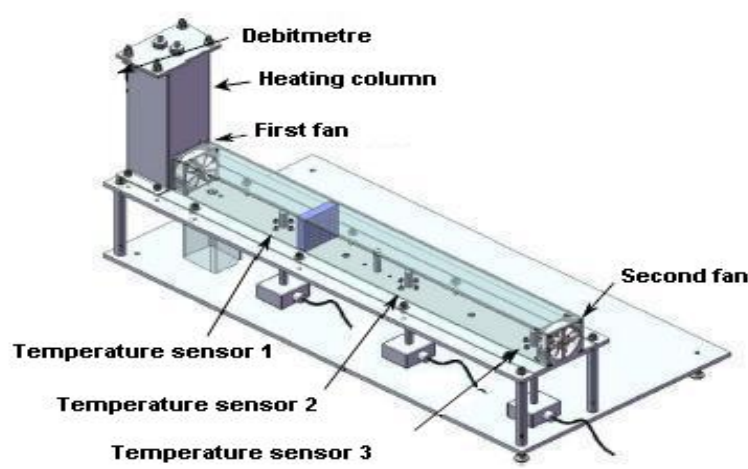

Fig. 1. Experimental Setup

This setup consists of two parts: a heating column and a tube. The gas dynamics in the heating column is governed by a finite dimensional ordinary differential equation. Whereas a partial differential equation is used to model the dynamics in the tube. Since the area of the cross section of the heating column is much larger than that of the tube, this leads to the gas velocity in the heating column being much slower than that in the tube. Thus we can model this setup by a singularly perturbed coupled ODE-PDE system due to two time scales' dynamics.

In the present paper we first consider an ODE coupled with a fast linear hyperbolic system, which can be used to model the above setup. The stability analysis shows that if the reduced and the boundary-layer subsystems are stable then the full system is stable. This is proved by a Lyapunov function. The simulations on the gas flow transport system illustrate the first result. Secondly, we study a fast ODE coupled with a hyperbolic PDE. An example indicates that the stability of both subsystems does not ensure the full system's stability, which is different from the previous system.

The paper is outlined as follows. Section II studies ODE coupled with fast PDE systems. Two subsystems are formally computed in the same section as well as the stability analysis between the full system and the subsystems, which is established by Lyapunov techniques. Section III shows the 
numerical solutions of the gas transport model. Section IV presents PDE coupled with fast ODE systems. An example is used to prove that the stability of both subsystems does not guarantee that of the full system. Finally, concluding remarks end the paper.

Notation. Given a matrix $M, M^{-1}$ and $M^{\top}$ represent the inverse and the transpose matrix of $M$ respectively. For a symmetric matrix, $\star$ denotes the symmetric part. The minimum eigenvalue of the matrix $M$ is denoted by $\underline{\lambda}(M)$. For a positive integer $n, I_{n}$ is the identity matrix in $\mathbb{R}^{n \times n} .||$ denotes the usual Euclidean norm in $\mathbb{R}^{n}$ and \|\| is associated with the matrix norm. \|\|$_{L^{2}}$ denotes the associated norm in $L^{2}(0,1)$ space, defined by $\|f\|_{L^{2}}=\left(\int_{0}^{1}|f(x)|^{2} d x\right)^{\frac{1}{2}}$ for all functions $f \in L^{2}(0,1)$. Following [8], we introduce the notation, for all matrices $K \in \mathbb{R}^{n \times n}$,

$$
\rho_{1}(K)=\inf \left\{\left\|\Delta K \Delta^{-1}\right\|, \Delta \in D_{n,+}\right\},
$$

where $D_{n,+}$ denotes the set of diagonal positive matrices in $\mathbb{R}^{n \times n}$.

\section{ODE COUPLED WITH FAST PDE}

Let us consider an ordinary differential system (ODE) coupled with a hyperbolic system described by a partial differential equation (PDE). A positive perturbation parameter $\varepsilon$ is introduced into the dynamics of the PDE system:

$$
\begin{aligned}
& \dot{Z}(t)=A Z(t)+B y(1, t), \\
& \varepsilon y_{t}(x, t)+\Lambda y_{x}(x, t)=0 .
\end{aligned}
$$

where $x \in[0,1], t \in[0,+\infty), Z:[0,+\infty) \rightarrow \mathbb{R}^{n}$, $y:[0,1] \times[0,+\infty) \rightarrow \mathbb{R}^{m}, \Lambda$ is a diagonal positive matrix in $\mathbb{R}^{m \times m}$. In (1a) $\mathrm{A}$ and $\mathrm{B}$ are matrices of appropriate dimensions. The boundary condition for system (1) is given by

$$
y(0, t)=G_{1} y(1, t)+G_{2} Z(t)
$$

where $G_{1}$ and $G_{2}$ are real constant matrices with appropriate dimension. The initial conditions are

$$
\begin{gathered}
Z(0)=Z_{0}, \\
y(x, 0)=y_{0}(x) .
\end{gathered}
$$

Remark 1: The existence and the uniqueness of the solution of such coupled system have been studied in many research work. For instance, according to Theorem A.6. in [1], for every $Z_{0} \in \mathbb{R}^{n}$, for every $y_{0} \in L_{2}(0,1)$, the Cauchy problem (1)-(3) has one and only one solution $Z \in C^{0}\left([0,+\infty), \mathbb{R}^{n}\right), y \in C^{0}\left([0,+\infty),\left(L^{2}(0,1), \mathbb{R}^{m}\right)\right)$. Since system (1) contains two time scales, let us decompose it into the slow and fast subsystems, namely the reduced and boundary-layer subsystems. Adopting the approach in [15], the two subsystems are formally computed as follows. By setting $\varepsilon=0$ in system (1b), we obtain

$$
y_{x}(x, t)=0 \text {. }
$$

It implies $y(., t)=y(1, t)$. Using this fact to the boundary condition (2) and assuming $\left(I_{m}-G_{1}\right)$ invertible, yield

$$
y(., t)=G_{r} Z(t),
$$

where $G_{r}=\left(I_{m}-G_{1}\right)^{-1} G_{2}$. Using the right hand side of (5) to replace $y(1, t)$ in $(1 \mathrm{a})$, the reduced subsystem is computed as follows,

$$
\dot{\bar{Z}}(t)=\left(A+B G_{r}\right) \bar{Z}(t)
$$

the initial condition is given by

$$
\bar{Z}_{0}=Z_{0}
$$

Performing the following change of variable

$$
\bar{y}=y-G_{r} Z
$$

which shifts the equilibrium of $y$ to the origin. The boundarylayer subsystem is computed in time scale $\tau=t / \varepsilon$

$$
\bar{y}_{\tau}(x, \tau)+\Lambda \bar{y}_{x}(x, \tau)=0
$$

with the boundary condition

$$
\bar{y}(0, \tau)=G_{1} \bar{y}(1, \tau), \quad \tau \in[0,+\infty) .
$$

The initial condition is given by

$$
\bar{y}_{0}=y_{0}-G_{r} Z_{0} .
$$

To state the stability of the full system (1)-(3), let us firstly present the following assumptions

Assumption 1: The matrix $A+B G_{r}$ is Hurwitz.

Assumption 2: The boundary condition $G_{1}$ satisfies $\rho_{1}\left(G_{1}\right)<1$.

Let us recall the following proposition for the stability of the linear hyperbolic system.

Proposition 1: [9] If $\rho_{1}\left(G_{1}\right)<1$, then the linear system (8)-(10) is exponentially stable in $L^{2}$-norm.

We are ready to state our main result in the following theorem.

Theorem 1: Under Assumptions 1-2, there exists $\varepsilon^{*}>$ 0, such that for all $\varepsilon \in\left(0, \varepsilon^{*}\right)$, the full system (1)-(3) is exponentially stable in $L^{2}$-norm, that is for any $Z_{0} \in \mathbb{R}^{n}$, $y_{0} \in L^{2}(0,1)$, there exist positive values $C$ and $\nu$, it holds for all $t \geqslant 0,\left(|Z(t)|^{2}+\|y(., t)\|_{L^{2}}^{2}\right) \leqslant C e^{-\nu t}\left(\left|Z_{0}\right|^{2}+\right.$ $\left.\left\|y_{0}\right\|_{L^{2}}^{2}\right)$. Moreover, it has a strict Lyapunov function

$V(Z, y)=Z^{\top} P Z+\int_{0}^{1} e^{-\mu x}\left(y-G_{r} Z\right)^{\top} Q\left(y-G_{r} Z\right) d x$,

where $\mu>0, P$ is a symmetric positive matrix and $Q$ is diagonal positive.

Proof: Let us consider $V$ defined by (11) and write it as $V=V_{1}+V_{2}$, with

$$
\begin{gathered}
V_{1}=Z^{\top} P Z \\
V_{2}=\int_{0}^{1} e^{-\mu x}\left(y-G_{r} Z\right)^{\top} Q\left(y-G_{r} Z\right) d x,
\end{gathered}
$$


where $P$ and $Q$ will be specified later. Computing the time derivative of $V_{1}$ along the solution to system (1a) yields

$$
\begin{aligned}
\dot{V}_{1}= & 2 Z^{\top} P \dot{Z} \\
= & 2 Z^{\top} P(A Z+B y(1)) \\
= & 2 Z^{\top} P\left(\left(A+B G_{r}\right) Z+B\left(y(1)-G_{r} Z\right)\right) \\
= & Z^{\top}\left(P\left(A+B G_{r}\right)+\left(A+B G_{r}\right)^{\top} P\right) Z \\
& +2 Z^{\top} P B\left(y(1)-G_{r} Z\right) .
\end{aligned}
$$

Under Assumption 1, there exists a symmetric positive matrix $P$ such that

$$
\left(A+B G_{r}\right)^{\top} P+P\left(A+B G_{r}\right)<-I_{n} .
$$

Due to Cauchy Schwarz inequality, it follows

$$
\dot{V}_{1} \leqslant-|Z|^{2}+2\|P B\|\left|y(1)-G_{r} Z\right||Z| .
$$

Similarly, computing the time derivative of $V_{2}$ along the solution to system (1b) yields

$$
\begin{aligned}
\dot{V}_{2}=2 \int_{0}^{1} e^{-\mu x}\left(y-G_{r} Z\right)^{\top} Q\left(y_{t}-G_{r} \dot{Z}\right) d x \\
=2 \int_{0}^{1} e^{-\mu x}\left(y-G_{r} Z\right)^{\top} Q\left(-\frac{\Lambda y_{x}}{\varepsilon}-G_{r}(A Z+B y(1))\right) d x \\
=-\frac{2}{\varepsilon} \int_{0}^{1} e^{-\mu x}\left(y-G_{r} Z\right)^{\top} Q \Lambda y_{x} d x \\
\quad-2 \int_{0}^{1} e^{-\mu x}\left(y-G_{r} Z\right)^{\top} Q G_{r}(A Z+B y(1)) d x .
\end{aligned}
$$

Performing an integration by parts on the first integral and reorganizing it, we can write $\dot{V}_{2}=V_{21}+V_{22}+V_{23}$ with

$V_{21}=\frac{\left[\left(y(1)-G_{r} Z\right)^{\top}\left(e^{-\mu} Q \Lambda-G_{1}^{\top} Q \Lambda G_{1}\right)\left(y(1)-G_{r} Z\right)\right]}{-\varepsilon}$,

$V_{22}=-\frac{\mu}{\varepsilon} \int_{0}^{1} e^{-\mu x}\left(y-G_{r} Z\right)^{\top} Q \Lambda\left(y-G_{r} Z\right) d x$,

$V_{23}=-2 \int_{0}^{1} e^{-\mu x}\left(y-G_{r} Z\right)^{\top} Q G_{r}(A Z+B y(1)) d x$.

Under Assumption 2, $\rho_{1}\left(G_{1}\right)<1$ implies that there exists a diagonal positive matrix $\Delta$ such that $\left\|\Delta G_{1} \Delta^{-1}\right\|<1$. It is equivalent to $\Delta^{2}-G_{1}^{\top} \Delta^{2} G_{1}>0$. Let choose $Q=\Delta^{2} \Lambda^{-1}$, for $\mu>0$ small enough, it holds

$$
e^{-\mu} Q \Lambda-G_{1}^{\top} Q \Lambda G_{1}>\underline{\lambda}\left(e^{-\mu} Q \Lambda-G_{1}^{\top} Q \Lambda G_{1}\right)>0 .
$$

Therefore

$$
V_{21} \leqslant-\frac{\lambda\left(e^{-\mu} Q \Lambda-G_{1}^{\top} Q \Lambda G_{1}\right)}{\varepsilon}\left|y(1)-G_{r} Z\right|^{2} .
$$

$V_{22}$ follows

$$
V_{22} \leqslant-\frac{\mu e^{-\mu} \underline{\lambda}(Q \Lambda)}{\varepsilon} \int_{0}^{1}\left|y-G_{r} Z\right|^{2} d x .
$$

The following is deduced from $V_{23}$

$$
\begin{aligned}
V_{23}= & -2 \int_{0}^{1} e^{-\mu x}\left(y-G_{r} Z\right)^{\top} Q G_{r}\left(A+B G_{r}\right) Z d x \\
& -2 \int_{0}^{1} e^{-\mu x}\left(y-G_{r} Z\right)^{\top} Q G_{r} B\left(y(1)-G_{r} Z\right) d x .
\end{aligned}
$$

Due to Cauchy Schwarz inequality and Young's inequality, $V_{23}$ follows

$$
\begin{gathered}
V_{23} \leqslant 2\left\|Q G_{r}\left(A+B G_{r}\right)\right\||Z| \int_{0}^{1}\left|y-G_{r} Z\right| d x \\
\quad+2\left\|Q G_{r} B\right\|\left|y(1)-G_{r} Z\right| \int_{0}^{1}\left|y-G_{r} Z\right| d x .
\end{gathered}
$$

Combining (13), (14) and (15) yields

$$
\begin{aligned}
\dot{V}_{2} \leqslant & -\frac{\lambda\left(e^{-\mu} Q \Lambda-G_{1}^{\top} Q \Lambda G_{1}\right)}{\varepsilon}\left|y(1)-G_{r} Z\right|^{2} \\
& -\frac{\mu e^{-\mu} \underline{\lambda}(Q \Lambda)}{\varepsilon}\left\|y-G_{r} Z\right\|^{2} \\
& +2\left\|Q G_{r}\left(A+B G_{r}\right)\right\||Z|\left\|y-G_{r} Z\right\| \\
& +2\left\|Q G_{r} B\right\|\left|y(1)-G_{r} Z\right|\left\|y-G_{r} Z\right\| .
\end{aligned}
$$

Combining (12) and (16), we obtain

$$
\dot{V} \leqslant-\left(\begin{array}{c}
\left|y(1)-G_{r} Z\right| \\
|Z| \\
\left\|y-G_{r} Z\right\|_{L^{2}}
\end{array}\right)^{\top} M\left(\begin{array}{c}
\left|y(1)-G_{r} Z\right| \\
|Z| \\
\left\|y-G_{r} Z\right\|_{L^{2}}
\end{array}\right)
$$

where $M=\left(\begin{array}{cc}M_{1} & M_{2} \\ \star & M_{4}\end{array}\right)$,

with $M_{1}=\left(\begin{array}{cc}M_{11} & M_{12} \\ \star & M_{14}\end{array}\right)=\left(\begin{array}{cc}\frac{\lambda\left(e^{-\mu} Q \Lambda-G_{1}^{\top} Q \Lambda G_{1}\right)}{\varepsilon} & \|P B\| \\ \star & 1\end{array}\right)$, $M_{2}=\left(\begin{array}{c}-\left\|Q G_{r} B\right\| \\ -\left\|Q G_{r}\left(A+B G_{r}\right)\right\|\end{array}\right), M_{4}=\left(\frac{\mu e^{-\mu} \frac{\lambda}{\underline{\lambda}(Q \Lambda)}}{\varepsilon}\right)$.

First, let us study the matrix $M_{1}$. Since $M_{14}>0$ and there exists $\varepsilon_{1}^{*}$ such that for $\varepsilon \in\left(0, \varepsilon_{1}^{*}\right), M_{11}-M_{12} M_{14}^{-1} M_{12}^{\top}>0$. Due to the Schur complement, we get $M_{1}>0$. The inverse of $M_{1}$ is computed as

$$
\begin{aligned}
M_{1}^{-1}= & \frac{1}{\underline{\lambda}\left(e^{-\mu} Q \Lambda-G_{1}^{\top} Q \Lambda G_{1}\right)-\varepsilon\|P B\|^{2}} \\
& \times\left(\begin{array}{cc}
\varepsilon & \varepsilon\|P B\| \\
\star \underline{\lambda}\left(e^{-\mu} Q \Lambda-G_{1}^{\top} Q \Lambda G_{1}\right)
\end{array}\right) .
\end{aligned}
$$

There exists $\varepsilon_{2}^{*}$, such that for all $0<\varepsilon<\min \left(\varepsilon_{1}^{*}, \varepsilon_{2}^{*}\right), M_{4}-$ $M_{2}^{\top} M_{1}^{-1} M_{2}>0$. We get $M>0$ according to the Schur complement. Thus there exists $\alpha>0$ such that

$$
\dot{V} \leqslant-\alpha V \text {. }
$$

This concludes the proof of Theorem 1 .

\section{NUMERICAL SIMULATIONS ON A GAS FLOW TRANSPORT MODEL}

Let us recall the experimental setup in Figure 1. It consists of two subsystems, the heating column and the tube. The two subsystems are modeled as follows.

Model of the heating column: To model the gas dynamics in the heating column, we first consider the following assumptions.

Assumption 3: The dynamics of the pressure in the gas control volume is much faster than that of the temperature, the pressure and the mass can be considered as quasi static. 
Assumption 4: The pressure losses are neglected because of the low mass flow and of the sufficiently large input/output section of gas. This implies $p_{0} \approx p_{i n}$, where $p_{\text {in }}$ is input pressure.

Assumption 5: There is no work done by gas.

Under the above three assumptions and due to the first law of thermodynamics and ideal gas law, the gas dynamics in the heating column is modeled by (see [3])

$$
\dot{\rho}_{0}=-\frac{R \gamma T_{i n} \dot{m}_{i n}}{p_{i n} V_{0}} \rho_{0}-\frac{R}{p_{i n} V_{0} C_{v}} \rho_{0} d Q+\frac{\gamma \dot{m}_{i n}}{V_{0}},
$$

where $\rho_{0}$ is the gas density in the heating column, $R$ is the specific gas constant, $T_{i n}$ denotes the gas temperature at input, the input mass flow is given by $\dot{m}_{i n}, V_{0}$ is the volume of the heating column, $C_{v}$ and $C_{p}$ are the special heat of volume constant gas and of pressure constant gas respectively, $d Q$ is the heating exchange that can be controlled and $\gamma=\frac{C_{p}}{C_{v}}$.

Model of the tube: To model the gas dynamics in the tube, let us state the following assumptions.

Assumption 6: All the heat transfers and friction losses are negligible.

Assumption 7: The gas pressure in the tube is assumed to be constant, which is close to the atmosphere pressure. Under Assumptions 6-7, the gas dynamics in the tube is given by (see [3]), for $x \in[0,1]$ and for $t \geqslant 0$,

$$
\rho_{t}(x, t)+u_{b} \rho_{x}(x, t)=0,
$$

where $\rho$ represents the gas density in the tube. The propagation speed in the tube is denoted by $u_{b}$. With a scaling of the space domain, it may be assumed that the tube's length equals 1 . Due to ideal gas law, $u_{b}=\frac{\dot{m}_{i n}}{\rho_{0} S_{b}}$, where $S_{b}$ is the cross section of the tube.

The boundary condition is given by

$$
\rho(0, t)=\rho_{0} .
$$

Control problem statement: In the following we state our control problem. Let us rewrite (17) and (18) as follows

$$
\begin{array}{r}
\dot{\rho}_{0}=-\frac{1}{\kappa} \rho_{0}+U(t), \\
\rho_{t}+u_{b} \rho_{x}=0 .
\end{array}
$$

where $\kappa$ is the transport time constant in the heating column and $U(t)$ is the control (it could be defined from $d Q$ ). The boundary condition is the same as (19).

The control problem is formulated as: for any desired mass density in the tube $\rho^{*} \geqslant 0$, let the controller be

$$
U(t)=c_{1} \rho(1, t)+c_{2} \rho^{*},
$$

such that the system is exponentially stable at the equilibrium point $\rho=\rho^{*}$ with an appropriate choice of real values $c_{1}$ and $c_{2}$. Replacing $U(t)$ in (20a) by the right hand side in (21), the closed-loop system is written as

$$
\begin{array}{r}
\dot{\rho}_{0}=-\frac{1}{\kappa} \rho_{0}+c_{1} \rho(1, t)+c_{2} \rho^{*}, \\
\rho_{t}+u_{b} \rho_{x}=0,
\end{array}
$$

with the same boundary condition (19).

At the equilibrium point of the gas density inside of the tube $\rho_{0}^{*}$, from (22a), we get $\rho^{*}=\frac{\frac{1}{k}-c_{1}}{c_{2}} \rho_{0}^{*}$, where $c_{2}$ has to be selected such that $c_{2} \neq 0$. Due to (19), it holds at the equilibrium $\rho^{*}=\rho_{0}^{*}$. Therefore, the values of $c_{1}$ and $c_{2}$ should satisfy $c_{1}+c_{2}=\frac{1}{\kappa}$. Let us define the state deviations with respect to the equilibrium point $\tilde{\rho}_{0}=\rho_{0}-\rho^{*}$, and $\tilde{\rho}=\rho-\rho^{*}$. The linearized system is

$$
\begin{array}{r}
\dot{\tilde{\rho}}_{0}=-\frac{1}{\kappa} \tilde{\rho}_{0}+c_{1} \tilde{\rho}(1, t), \\
\varepsilon \tilde{\rho}_{t}+\frac{1}{\kappa} \tilde{\rho}_{x}=0,
\end{array}
$$

where the perturbation parameter is given by $\varepsilon=\frac{1 / \kappa}{u_{b}^{*}}$, due to the transport velocity of gas in the heating column is much smaller than that in the tube. The boundary condition is

$$
\tilde{\rho}(0, t)=\tilde{\rho}_{0} .
$$

From (24), recalling the condition in (2), we compute $G_{1}=$ $0, G_{2}=1$ and $G_{r}=1$. Moreover, by (1a) and (6), Assumption 1 holds as soon as $c_{1}<\frac{1}{\kappa}$. Assumption 2 holds since $\rho_{1}\left(G_{1}\right)=0$.

The reduced subsystem is

$$
\dot{\bar{\rho}}_{0}=\left(-\frac{1}{\kappa}+c_{1}\right) \bar{\rho}_{0},
$$

whereas the boundary-layer subsystem is written in time scale $\tau=\frac{t}{\varepsilon}$

$$
\bar{\rho}_{\tau}+\frac{1}{\kappa} \bar{\rho}_{x}=0,
$$

with the boundary condition

$$
\bar{\rho}(0, \tau)=0 .
$$

Let us take the experimental data from [3]: $\gamma=1.4$, $R=8.3 \mathrm{~J} /(\mathrm{mol} * \mathrm{~K}), p_{\text {in }}=1 \times 10^{5} \mathrm{~Pa}, T_{\text {in }}=300 \mathrm{~K}$, $V_{0}=4 \times 10^{-3} \mathrm{~m}^{3}, \dot{m}_{i n}=0.01 \mathrm{~kg} / \mathrm{s}, S_{b}=6.4 \times 10^{-3}$. We compute $\kappa=10, \varepsilon=0.1$. We choose $c_{1}=0.01$. The initial conditions are given by: $\rho_{0}(0)=\bar{\rho}_{0}(0)=2$, $\rho(0)=\cos (4 \pi x)-1, \bar{\rho}(0)=\rho(0)-\rho_{0}(0)=\cos (4 \pi x)-3$. Let us use a two-step variant of the Lax-Wendroff method (see [20] and [21]) to check the numerical solutions. The solution of the boundary-layer subsystem is shown in Figure 2 , it converges to the origin. Figure 3 presents the solutions of the reduced subsystem and the slow dynamics of the full system, which decrease to zero as time increases and the evolution of the two curves is roughly the same. In Figure 4 , it is shown that the solution of the fast dynamics of the full system tends to zero as time increases, as expected from Theorem 1.

\section{PDE COUPLED WITH FAST ODE}

In this section let us consider a PDE coupled with an ODE, where the perturbation parameter is introduced into the dynamics of the ODE. The full system is given by

$$
\begin{aligned}
& \varepsilon \dot{Y}(t)=C Y(t)+D z(1), \\
& z_{t}(x, t)+\Lambda_{1} z_{x}(x, t)=0,
\end{aligned}
$$




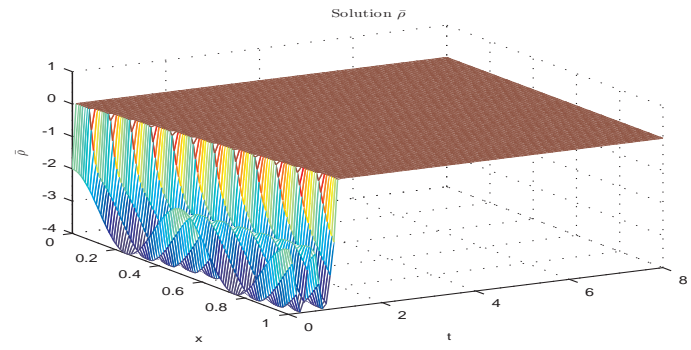

Fig. 2. Solution of the boundary-layer subsystem (26)-(27)

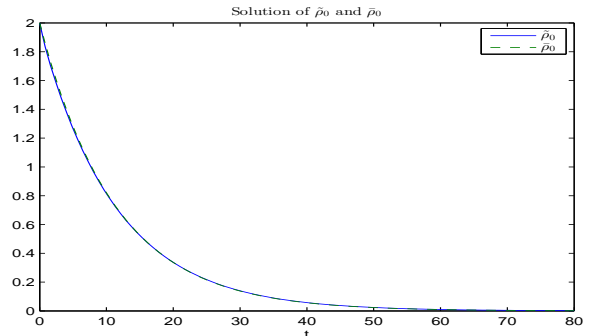

Fig. 3. Solutions of the reduced subsystem (25)(dashed line) and the slow dynamics of the full system (23)-(24)(plain line)

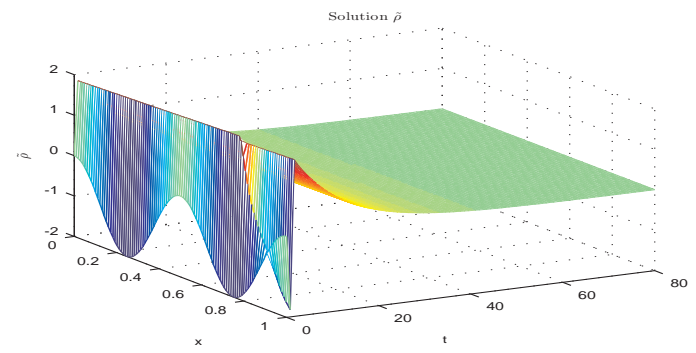

Fig. 4. Solution of the fast dynamics of the full system (23)-(24)

where $x \in[0,1], t \in[0,+\infty), Y:[0,+\infty) \rightarrow \mathbb{R}^{n}, z:$ $[0,1] \times[0,+\infty) \rightarrow \mathbb{R}^{m}, \Lambda_{1}$ is a diagonal positive matrix in $\mathbb{R}^{m \times m}$. In (28a) $\mathrm{C}$ and $\mathrm{D}$ are matrices with appropriate dimension. The boundary condition is given by

$$
z(0, t)=K_{1} z(1, t)+K_{2} Y(t),
$$

where $K_{1}$ and $K_{2}$ are in appropriate dimension. The initial conditions are

$$
\begin{gathered}
Y(0)=Y_{0}, \\
z(x, 0)=z_{0}(x) .
\end{gathered}
$$

The reduced subsystem is computed as

$$
\bar{z}_{t}(x, t)+\Lambda_{1} \bar{z}_{x}(x, t)=0,
$$

with the boundary condition

$$
\bar{z}(0, t)=K_{r} \bar{z}(1, t),
$$

where $K_{r}=\left(K_{1}-K_{2} C^{-1} D\right)$, whereas the initial condition is

$$
\bar{z}(x, 0)=\bar{z}_{0}(x)=z_{0}(x)
$$

To ensure the existence of a solution of the reduced subsystem in $H^{2}$, the compatibility conditions are given by

$$
\begin{aligned}
\bar{z}_{0}(0) & =K_{r} \bar{z}_{0}(1), \\
\bar{z}_{0_{x}}(0) & =\Lambda^{-1} K_{r} \Lambda \bar{z}_{0_{x}}(1) .
\end{aligned}
$$

Perform a change of variable $\bar{Y}=Y+C^{-1} D z(1)$, the boundary-layer subsystem is computed as

$$
\frac{d \bar{Y}(\tau)}{d \tau}=C \bar{Y}(\tau)
$$

with the time scale $\tau=t / \varepsilon$. The initial condition is given by

$$
\bar{Y}(0)=\bar{Y}_{0}
$$

The assumptions, which are used in this section, are given as follows

Assumption 8: The boundary condition $K_{r}$ satisfies $\rho_{1}\left(K_{r}\right)<1$

Assumption 9: The matrix $C$ is Hurwitz.

Let us perform a change of variables

$$
\begin{aligned}
\eta & =z-\bar{z} \\
\delta & =Y+C^{-1} D \bar{z}(1)-\bar{Y}(t / \varepsilon) .
\end{aligned}
$$

The system in variable $(\eta, \delta)$ are computed as follows

$$
\begin{aligned}
& \eta_{t}+\Lambda_{1} \eta_{x}=0, \\
& \eta(0, t)=K_{1} \eta(1, t)+K_{2} \delta(t)+K_{2} \bar{Y}(t / \varepsilon), \\
& \varepsilon \dot{\delta}(t)=C \delta-\varepsilon C^{-1} D \Lambda_{1} \bar{z}_{x}(1, t) .
\end{aligned}
$$

Note that (41) is well defined since $\bar{z}(x, t) \in H^{2}$. Surprisingly, Theorem 1 is not valid in the context of this section. To be more precise, it holds Proposition 2.

Proposition 2: Assumptions 8 and 9 do not imply the exponential stability of system (28)-(29).

We provide an example to prove the above proposition. Proof: We consider the following $2 \times 2$ system

$$
\begin{gathered}
\varepsilon \dot{Y}(t)=-0.1 Y(t)-z(1), \\
z_{t}(x, t)+z_{x}(x, t)=0 .
\end{gathered}
$$

The boundary condition is given by $K_{1}=2$ and $K_{2}=0.2$,

$$
z(0, t)=2 z(1, t)+0.2 Y(t) .
$$

The initial conditions are chosen as $z_{0}=0$ and $Y_{0} \neq 0$.

The reduced subsystem is computed as

$$
\bar{z}_{t}(x, t)+\bar{z}_{x}(x, t)=0,
$$

with the boundary condition

$$
\bar{z}(0, t)=0 .
$$

The initial condition is chosen as $\bar{z}_{0}=z_{0}=0$.

The explicit solution of (44)-(45) is

$$
\bar{z}(x, t)=0 .
$$

The boundary-layer subsystem is computed as

$$
\frac{d \bar{Y}(\tau)}{d \tau}=-0.1 \bar{Y}(\tau)
$$


The solution of (46) is

$$
\bar{Y}(t / \varepsilon)=e^{-0.1 t / \varepsilon} \bar{Y}_{0} .
$$

System (41) is written as

$$
\dot{\delta}(t)=-\frac{0.1}{\varepsilon} \delta(t)
$$

The solution of (48) is

$$
\delta(t)=e^{-0.1 t / \varepsilon} \delta_{0}
$$

Due to (38), we obtain $\delta_{0}=0$. Thus $\delta(t)=0$ for all $t \geqslant 0$. System (39)-(40) is written as

$$
\begin{gathered}
\eta_{t}+\eta_{x}=0 \\
\eta(0, t)=2 \eta(1, t)+0.2 \bar{Y}(t / \varepsilon) .
\end{gathered}
$$

Let us denote $f(t)=0.2 \bar{Y}(t / \varepsilon)$, we can compute for all $t \geqslant 0$

$$
\eta(1, t)=2 \eta(1, t-1)+f(t-1)
$$

Using a recursion, it can be proved that for all $t \in \mathbb{N}$,

$$
\eta(1, t)=2^{t-1} \sum_{s=0}^{t-1} f(s) 2^{-s}+2^{t} \eta(1,0) .
$$

Since $\eta(1,0)=z_{0}(1)-\bar{z}_{0}(1)=0, \bar{Y}_{0}=Y_{0}$ and using (47), it follows for all $t \in \mathbb{N}$,

$$
\eta(1, t)=2^{t-1} \times 0.2 Y_{0} \sum_{s=0}^{t-1} 2^{-s} e^{-0.1 s / \varepsilon} .
$$

Since the initial condition $Y_{0}$ is chosen as $Y_{0} \neq 0$, then $\eta(1, t)$ tends to infinity. Since $\bar{z}(x, t)=0$, thus $z(1, t)=$ $\eta(1, t)$ diverges. Then it can be shown that the full system (42)-(43) is unstable in $L^{2}$-norm. This example illustrates that the stability of both subsystems does not ensure the stability of the full system as stated in Proposition 2.

\section{CONCLUSION}

This paper has dealt with a coupled ODE-PDE system with two time scales. In the first case, it has been considered ODE coupled with fast PDE systems. It has been shown that the stability of the reduced and the boundary-layer subsystems ensures the stability of the full system. This has been proved by a strict Lyapunov function. The application to a gas flow transport model governed by an ODE-PDE system has been used to illustrate the main result. In the second case, it has been considered PDE coupled with fast ODE systems. An example has been provided to prove that the full system's stability cannot be guaranteed by that of both subsystems.

\section{REFERENCES}

[1] G. Bastin and J-M. Coron. Stability and Boundary Stabilization of 1-D Hyperbolic Systems. Preprint, 2015.

[2] G. Bastin, J-M. Coron, and S.O. Tamasoiu. Stability of linear densityflow hyperbolic systems under PI boundary control. Automatica, 53:37-42, 2015.

[3] F. Castillo, E. Witrant, and L. Dugard. Contrôle de température dans un flux de Poiseuille. In IEEE Conférence Internationale Francophone d'Automatique, Grenoble, France, 2012.

[4] J.H. Chow. Asymptotic stability of a class of nonlinear singularly perturbed systems. Journal of The Franklin Institute, 305(5):275-281, 1978.

[5] J.H. Chow and P.V. Kokotović. A two-stage Lyapunov-Bellman feedback design of a class of nonlinear systems. IEEE Transactions on Automatic Control, 26(3):656-663, 1981.

[6] R.M. Colombo, G. Guerra, M. Herty, and V. Schleper. Optimal control in networks of pipes and canals. SIAM Journal on Control and Optimization, 48:2032-2050, 2009.

[7] J-M. Coron, G. Bastin, and B. d'Andréa-Novel. A strict Lyapunov function for boundary control of hyperbolic systems of conservation laws. IEEE Transactions on Automatic Control, 52:2-11, 2007.

[8] J-M. Coron, G. Bastin, and B. d'Andréa-Novel. Dissipative boundary conditions for one-dimensional nonlinear hyperbolic systems. SIAM Journal on Control and Optimization, 47(3):1460-1498, 2008.

[9] A. Diagne, G. Bastin, and J-M. Coron. Lyapunov exponential stability of 1-D linear hyperbolic systems of balance laws. Automatica, 48:109114, 2012.

[10] M. Dick, M. Gugat, and G. Leugering. Classical solutions and feedback stabilization for the gas flow in a sequence of pipes. Networks and Heterogeneous Media, 5(4):691-709, 2010.

[11] V. Dos Santos, G. Bastin, J-M. Coron, and B. d'Andréa-Novel. Boundary control with integral action for hyperbolic systems of conservation laws: Stability and expriments. Automatica, 44:1013-1318, 2008.

[12] L.T. Grujic. Uniform asymptotic stability of nonlinear singularly perturbed and large scale systems. International Journal of Control, 33(3):481-504, 1981

[13] M. Gugat, G. Leugering, S. Tamasoiu, and K. Wang. $\mathrm{H}^{2}$ stabilization of the isothermal Euler equations: a Lyapunov function approach. Chinese Annals of Mathematics, Series B, 33:479-500, 2012.

[14] P. Habets. Stabilité asymptotique pour des problèmes de perturbations singulières. Centro Internazionalle Matematico Estimo, 1974.

[15] P. Kokotović, H.K. Khalil, and J. O'Reilly. Singular perturbation methods in control: analysis and design. Academic Press, 1986.

[16] M. Krstic. Compensating a string PDE in the actuation or sensing path of an unstable ODE. IEEE Transactions on Automatic Control, 54(6):1362-1368, 2009.

[17] M. Krstic. Compensating actuator and sensor dynamics governed by diffusion PDEs. Systems \& Control Letters, 58:372-377, 2009.

[18] M. Krstic and A. Smyshlyaev. Backtepping boundary control for firstorder hyperbolic PDEs and application to systems with actuator and sensor delays. Systems \& Control Letters, 57:750-758, 2008.

[19] A. Saberi and H.K. Khalil. Quadratic-type Lyapunov functions for singularly perturbed systems. IEEE Transactions on Automatic Control, AC-29(6):542-550, 1984.

[20] L.F. Shampine. Solving hyperbolic PDEs in Matlab. Applied Numerical Analysis and Computational Mathematics, 2:346-358, 2005.

[21] L.F. Shampine. Two-step Lax-Friedrichs method. Applied Mathematics Letters, 18:1134-1136, 2005.

[22] Y. Tang. Stability analysis and Tikhonov approximation for linear singularly perturbed hyperbolic systems. $\mathrm{PhD}$ thesis, Univercité de Grenoble, 2015.

[23] Y. Tang, C. Prieur, and A. Girard. Tikhonov theorem for linear hyperbolic systems. Automatica, 57:1-10, 2015.

[24] J. Wang. Air fraction estimation for multiple combustion mode diesel engines with dual-loop EGR systems. Conrol Engine Practice, 16(1468-1479), 2008.

[25] E. Witrant, K. Johansson, and the HynX team. Air flow modelling in deep wells: application to mining ventilation. In IEEE Conference on Automation Science and Engineering, pages 845-850, Arlington, VA, 2008. 